tion of a product. In such cases, the FDA does not require the brand company to conduct full-scale clinical trials to confirm safety and efficacy.

A robust and reproducible process that yields a final product that consistently matches the desired composition of the reference product should be acceptable for the manufacturing of safe, effective and equivalent generic biopharmaceuticals.

With more than two decades of experience with many brand biopharmaceuticals, our knowledge of the causes of immunogenicity for specific products has increased significantly. Analytical testing of biopharmaceuticals can be used to minimize the potential for changes in the immunogenicity of a specific product.

The time has come. The substantial savings resulting from the entrance into the market of generic biopharmaceuticals will only be fully realized when a process is formalized that results in the approval of a generic product that can be directly substituted for the brandname version. For this reason, the US Congress and FDA must create a regulatory process that will help make biopharmaceuticals available and affordable for all by enabling the timely, efficient and cost-effective approval of generic versions of biopharmaceuticals.

The principles supporting the creation of an abbreviated approval pathway are clear. It must be based on sound scientific rationale that does not require unnecessary trials that have previously been conducted by the innovator. Clinical study requirements must be directly related to the complexity of the molecule. For those biopharmaceuticals that are less complex, characterization must be the cornerstone for approval. For moderately complex biopharmaceuticals, clinical studies must be limited, and trials should only be as large as necessary to scientifically demonstrate comparability of the generic to the brand-name product.

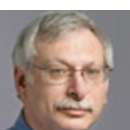

\section{COUNTERPOINT: Why biogenerics are a strawman}

\author{
Rob Garnick
}

US state and federal lawmakers are under the dual public policy pressures of both ensuring patient access to cheaper drugs and providing incentives for research companies to continue to innovate and invest resources in the search for breakthrough medical therapies. In trying to balance these goals, they must also be mindful that above all, individuals should have access to medicines that are safe and effective, not just inexpensive.

Several policy proposals have been suggested that seek to make drugs and drug development less expensive, possibly at the expense of safety and efficacy. The debate over 'follow-on' or 'generic' biologics is one suggested solution that would not necessarily result in less expensive drugs, and could put public safety at greater risk.

Beware of oversimplification. For some, the solution is simple: develop an approval pathway for generic or follow-on versions of biopharmaceuticals to obtain savings like those achieved with generic drugs. Although this solution may appear obvious and straightforward; in fact, it is far more

Rob Garnick, is Senior Vice President,

Regulatory, Quality and Compliance,

Genentech, 1 DNA Way, South San Francisco,

CA 94080, USA.

e-mail: snyder.robin@gene.com complicated than what some may think. The very nature of biopharmaceuticals raises serious scientific and legal questions regarding the validity and advisability of moving forward with an approval pathway for follow-on biotech products. The FDA should not proceed with developing such a pathway until these crucial questions are answered.

Biotech-derived products are made through intricate manufacturing processes that depend on living organisms (i.e., highly characterized cell lines). Unlike traditional pharmaceuticals, good scientific practice does not allow the direct comparison of one biotech product to another. This is because complex operational and proprietary details of the biotech manufacturing process are central to, and define the identity and unique structural characteristics of, each biotechderived product.

Accordingly, such processes are integral in determining the safety and effectiveness of those products. Because each biopharmaceutical manufacturing process is necessarily unique, and because small changes to any such process can have profound consequences on the end product, the FDA cannot rely on the analytical data generated from one biotech product to support approval of a product manufactured using another cell line and with different proprietary processes.
Small versus large molecules. Traditional small-molecule pharmaceuticals, on the other hand, are not made in living cells, but instead are made using highly reproducible processes involving chemical analysis. As such, small-molecule drugs can be defined completely by their atomic structure rather than by their manufacturing processes. In addition, a small-molecule drug can be shown, through chemical analysis, to be the same as another, fully analyzed and approved small-molecule product. As such, the safety and efficacy data generated for a small molecule by the innovator is relevant and applicable to the generic product. Because the products can be shown to be the same, the FDA can approve the generic product, without significant additional testing requirements and with confidence that the generic product is safe and effective.

Given this fundamental difference between biopharmaceuticals and more traditional, small-molecule drugs, the current paradigm for reviewing and approving generics is simply not applicable. As safety and efficacy data are not transferable between biopharmaceutical products, there can be no assurance that the follow-on product is safe or effective, as in the smallmolecule example.

Why we're not ready. The fact that biomanufacturing processes profoundly affect biotech products presents several problems to any abbreviated process for producing generic biopharmaceuticals. First, the proposed generic or follow-on product would be manufactured using an entirely different cell line, plasmid and process, as these materials and information belong to the biotech company and are closely guarded proprietary materials and trade secrets.

Second, to make effective comparisons between products would require that the FDA rely on a company's trade-secret manufacturing data. Were the FDA allowed to rely on a company's confidential and proprietary manufacturing data to the direct benefit of a competitor, there would be little incentive for a brand manufacturer to continue to invest the time and resources necessary to bringing breakthrough products to market.

As we continue to debate and consider approaches to providing patents with less expensive yet more effective and safe drugs, we must not rush toward solutions that appear expedient, yet are unsound scientifically and economically, and that could provide disincentives for innovators to continue to invest in discovering and developing new cures. 